\title{
ACY1 regulating PTEN/PI3K/AKT signaling in the promotion of non-small cell lung cancer progression
}

\author{
Hong Chen ${ }^{1 \#}$, Wei Wang ${ }^{2 \#}$, Caizhi Xiao ${ }^{1}$, Dongqin Xia ${ }^{1}$, Fangfei Li $^{1}$, Shaoyong Liu ${ }^{1}$ \\ ${ }^{1}$ Chongqing Key Laboratory of Translational Research for Cancer Metastasis and Individualized Treatment, Chongqing University Cancer Hospital \\ \& Chongqing Cancer Institute \& Chongqing Cancer Hospital, Chongqing, China; ${ }^{2}$ Key Laboratory for Biorheological Science and Technology \\ of Ministry of Education (Chongqing University), Chongqing University Cancer Hospital \& Chongqing Cancer Institute \& Chongqing Cancer \\ Hospital, Chongqing, China \\ Contributions: (I) Conception and design: H Chen, W Wang; (II) Administrative support: H Chen, W Wang; (III) Provision of study materials \\ or patients: C Xiao, D Xia; (IV) Collection and assembly of data: H Chen, W Wang, F Li, S Liu; (V) Data analysis and interpretation: H Chen, \\ W Wang, C Xiao, D Xia; (VI) Manuscript writing: All authors; (VII) Final approval of manuscript: All authors. \\ \#These authors contributed equally to this work. \\ Correspondence to: Wei Wang. Key Laboratory for Biorheological Science and Technology of Ministry of Education (Chongqing University), \\ Chongqing University Cancer Hospital \& Chongqing Cancer Institute \& Chongqing Cancer Hospital, 181 Hanyu Road, Chongqing 400044, \\ China. Email: wangweixiayanai@163.com.
}

Background: Non-small cell lung cancer (NSCLC) has a poor prognosis and is the most common cause of cancer-related deaths worldwide. Aminoacylase 1 (ACY1) plays a promoting role in some cancers, but its role in NSCLC is still unclear.

Methods: Immunohistochemistry, Reverse transcription-polymerase chain reaction (RT-PCR) and western blotting assays were used to determine ACY1 expression patterns in NSCLC tissues and cell lines. The clinical significance of ACY1 in NSCLC was evaluated by $\chi^{2}$ test and Kaplan-Meier analysis. MTT, flow cytometry, wound healing, and Transwell assays were performed to assess cell growth, apoptosis, migration, invasion, and tumorigenesis under different treatments. Male athymic BALB/C nude mice were used for xenotransplantation experiments.

Results: The results showed that ACY1 expression was elevated in NSCLC tissue samples and cells, and high ACY1 expression predicted an advanced clinical process and shorter overall survival in patients with NSCLC. Overexpression of ACY1 significantly increased cell growth, migration, invasion, and tumorigenesis, and reduced cell apoptosis, indicating that ACY1 functions as an oncogene in NSCLC. Moreover, ACY1 decreased phosphatase and tensin homolog (PTEN) expression, increased its ubiquitination, and activated PI3K/AKT signaling. Overexpression of PTEN diminished the effects of ACY1 upregulation on cell tumorigenesis promotion.

Conclusions: This study reveals that ACY1 may promote the progression of NSCLC via activating PI3K/AKT signaling in a PTEN-dependent manner. Our study may provide a better understanding of the pathogenesis and development of NSCLC.

Keywords: Aminoacylase 1 (ACY1); phosphatase and tensin homolog (PTEN); PI3K/AKT; non-small cell lung cancer (NSCLC)

Submitted May 30, 2021. Accepted for publication Aug 16, 2021.

doi: $10.21037 /$ atm-21-3127

View this article at: https://dx.doi.org/10.21037/atm-21-3127 


\section{Introduction}

Non-small cell lung cancer (NSCLC), including adenocarcinoma and squamous cell carcinoma, accounts for $80-85 \%$ of all lung cancer cases and is the most common cause of cancer-related deaths globally $(1,2)$. NSCLC has been identified as a highly heterogeneous type of cancer, harboring multiple genetic aberrations within its various subtypes (3). Despite recent advances in clinical and experimental oncology, the prognosis of lung cancer remains poor. Approximately $35 \%$ of NSCLC cases are diagnosed at stage IV, resulting in an overall 5 -year survival rate of only $15 \%(1,4)$. Therefore, further understanding of the mechanisms related to NSCLC progression is needed to develop novel therapeutic strategies.

Aminoacylase 1 (ACY1) is a homodimeric zinc-binding metalloenzyme which is widely expressed in mammalian tissues. It catalyzes the hydrolysis of $\mathrm{N}^{\mathrm{a}}$-acylated amino acids to L-amino acids and an acyl group (5). Recent studies have reported the role of ACY1 in tumorigenesis in several types of human cancer. For example, Miller et al. (6) found that ACY1 expression was reduced in a significant number of small-cell lung cancer (SCLC) cell lines. Additionally, Hwa and Balabanov et al. $(7,8)$ reported low ACY1 expression in renal clear cell carcinoma. Wei et al. (9) revealed that the expression of ACY1 was decreased in hepatocellular carcinoma (HCC) tissues and that it functioned as a tumor suppressor. However, ACY1 was reported to be overexpressed in colorectal cancer (CRC), and knockdown of ACY1 significantly inhibited proliferation and induced cell apoptosis $(10,11)$. It is showed that in neuroblastoma, ACY1 has the effect of inhibiting proliferation and metastasis (12). Nevertheless, the roles of ACY1 in NSCLC remain unclear.

The PI3K/AKT/mTOR pathway is a well-studied signal pathway which plays a key role in the modulation of many cellular functions, such as cell proliferation, apoptosis, metastasis, and invasion $(13,14)$. The hyperactivation of PI3K/AKT/mTOR can be detected in most cancers including NSCLC and is associated with cancer progression (15). Phosphatase and tensin homolog (PTEN), a tumor suppressor, has been reported to induce the dephosphorylation of the phospholipid products of $\mathrm{PI} 3 \mathrm{~K}$, thereby resulting in the repression of PI3K/AKT/ mTOR signaling $(15,16)$. Studies have confirmed that PTEN expression is regulated by number of genes which exert oncogenic or suppressive roles in the development of NSCLC, such as long noncoding RNA growth arrestspecific 5 (GAS5) (17), transforming growth factor beta $(T G F-\beta)(18)$, and tumor protein 53 target gene 1 (TP53TG1) (19).

Although the expression pattern of ACY1 has been found to be reduced in SCLC (6), to our knowledge, its function and clinical significance in NSCLC has not been clarified. In this study, we aimed to determine the expression profile of ACY1 in NSCLC, to evaluate the association between ACY1 expression and the clinical process and prognosis of patients with NSCLC, and to investigate whether PTEN/ $\mathrm{PI} 3 \mathrm{~K} / \mathrm{AKT} / \mathrm{mTOR}$ signaling is involved in vitro and in vivo. It is hoped that the findings of this study can aid in the development of new methods for the diagnosis and treatment of NSCLC.

We present the following article in accordance with the ARRIVE reporting checklist (available at https://dx.doi. org/10.21037/atm-21-3127).

\section{Methods}

\section{Tissue samples}

Twenty paired NSCLC and the adjacent normal tissue samples were obtained from patients with NSCLC who underwent pneumonectomy and did not receive any form of chemoradiotherapy. All tissue samples were stored at $-80^{\circ} \mathrm{C}$ immediately after resection. All procedures performed in this study involving human participants were in accordance with the Declaration of Helsinki (as revised in 2013). The study was approved by Ethics Committee of Chongqing University Cancer Hospital \& Chongqing Cancer Institute \& Chongqing Cancer Hospital (Chongqing, China) and informed consent was taken from all the patients.

\section{Immunobistochemistry}

First, the tissues were cut into $4-\mu \mathrm{m}$ slides. After deparaffinization and treatment to remove the endogenous peroxidase activity, antigen retrieval, and blocking with goat serum, the sections were incubated with the primary antiACY1 antibody (no. AF2900, R\&D Systems, Minneapolis, MA, USA) at a 1:100 dilution. The corresponding secondary antibody (Zhongshanqinqiao, Beijing, China) was then added and counterstained with Harris hematoxylin. Cell nuclei were dyed with 3,3'-diaminobenzidine (DAB; Serva, Heidelberg, Germany) at a 1:5,000 dilution. 
Table 1 Primer sequence

\begin{tabular}{ll}
\hline Genes & Sequence \\
\hline ACY1 & sense-5'-CAGCGACAGGAGAGTGAGC-3' \\
ACY1 & antisense-5'-CACATCCGTGTGGGAGTTGA-3 \\
PI3K & sense-5'-AAATGCTTGGGGTGGAAGGG-3' \\
PI3K & antisense-5'-ATGTATTCAGTTCAATTGCAGAAGG-3' \\
AKT & sense-5'-GAAGGACGGGAGCAGGC-3' \\
AKT & antisense-5'-TGTACTCCCCTCGTTTGTGC-3' \\
mTOR & sense-5'-CTTAGAGGACAGCGGGGAAG-3' \\
mTOR & antisense-5'-TCCAAGCATCTTGCCCTGAG-3' \\
PTEN & sense-5'-CAGCAGCTTCTGCCATCTCT-3' \\
PTEN & antisense-5'-TGCTTTGAATCCAAAAACCTTACT-3' \\
GAPDH & sense-5'-CCACTAGGCGCTCACTGTTCT-3' \\
GAPDH & antisense-5'-GCATCGCCCCACTTGATTTT-3' \\
\hline ACY1,
\end{tabular}

ACY1, aminoacylase 1; PI3K, phosphatidylinositol 3 kinase; AKT, protein kinase B; mTOR, mammalian target of rapamycin; PTEN, phosphatase and tensin homolog deleted on chromosome ten; GAPDH, glyceraldehyde-3-phosphate dehydrogenase.

\section{Cells culture}

Human embryonic lung WI-38 cells, along with NSCLC cell lines HCC2935, A549, and H1975, were obtained from the American Type Culture Collection (ATCC, VA, USA). WI-38 cells were grown in Eagle's Minimum Essential Medium; A549 cells in F-12K medium; and HCC2935 and H1975 cells in RPMI-1640 medium, supplemented with $10 \%$ fetal bovine serum (FBS) and $1 \%$ penicillin/ streptomycin. All reagents used for cell culture were obtained from Thermo Fisher Scientific (Danvers, MA, USA).

\section{Cell transfection}

The lentivirus vector shRNA-ACY1 used to silence ACY1 expression in NSCLC cells and its negative control (shNC) were purchased from OriGene (Beijing, China; no. TL315053). The overexpressing lentivirus vectors used to upregulate PTEN (OE-PTEN) and ACY1 (OE-ACY1) were obtained from GenePharma (Shanghai, China). Firstly, the gene fragments were amplified, and the amplified fragments were connected to the lentivirus and packaged.
To establish stable sh-ACY1- or OE-ACY1-transfected cell lines, H1975 cells were transfected with sh-ACY1 or OE-ACY1and then cultured with $8 \mu \mathrm{g} / \mathrm{mL}$ puromycin for 2 weeks, with the medium being changed every 2 days. Meanwhile, $100 \mu \mathrm{g} / \mathrm{mL}$ of G418 was used to select cells with OE-PTEN transfection.

\section{Real time quantitative polymerase chain reaction assay}

RNA was extracted from cells and tissues with an Oligotex mRNA Mini Lit (Qiagen, Valencia, CA, USA). The RNA was then reverse-transcribed into complement DNA (cDNA) with a PrimerScript RT reagent kit (TransGen Biotech, Beijing, China) in accordance with the instructions. Polymerase chain reaction (PCR) amplification was conducted using a SYBR Premix Ex Taq ${ }^{\mathrm{TM}}$ II kit (TransGen Biotech, Beijing, China) in a ViiATM 7 realtime fluorescence quantitative PCR system. Expression of messenger RNA (mRNA) was normalized to that of GAPDH. The relative expressions of mRNA were analyzed with the 2- $\Delta \Delta C T$ method. Primer sequences were showed int Table 1.

\section{Western blotting assay}

Cells in different groups and tissues were lysed with RIPA buffer (Thermo Scientific) at $4{ }^{\circ} \mathrm{C}$ for 30 minutes. Proteins were extracted and separated by $10 \%$ SDS-polyacrylamide gel electrophoresis and transferred onto a polyvinylidene fluoride (PVDF) membrane by electroblotting. Nonfat milk was used to block the PVDF membrane at $4{ }^{\circ} \mathrm{C}$ overnight. The membrane was then incubated with the primary antibodies at $4{ }^{\circ} \mathrm{C}$ overnight. The antibodies were showed in Table 2. The membranes were then incubated at room temperature for 1.5 hours with the secondary antibody $(1: 2,000$, Abcam). GAPDH was used as the internal reference. Target bands of proteins were visualized with an efficient chemiluminescence (ECL) western blotting kit (Millipore, Boston, MA, USA). Relative expression of proteins was assessed with the $2^{-\Delta \triangle C T}$ method.

\section{Immunoprecipitation assay}

NSCLC cells were treated with sh-NC, sh-ACY1, OE$\mathrm{NC}$, or OE-ACY1 for 48 hours before being rinsed with cold phosphate-buffered saline (PBS) and lysed in immunoprecipitation (IP) lysis buffer (Thermo Fisher Scientific). The cell lysate containing $200 \mu \mathrm{g}$ proteins 
Table 2 Antibodies

\begin{tabular}{lccl}
\hline Names & Dilution & Item No. & Company \\
\hline ACY1 & $1: 500$ & AF2900 & R\&D Systems, MN, USA \\
PI3K & $1: 500$ & 4252 & Cell Signaling Technology, Beverly, CA, USA \\
p-PI3K & $1: 500$ & 4228 & Cell Signaling Technology, Beverly, CA, USA \\
AKT & $1: 500$ & 9272 & Cell Signaling Technology, Beverly, CA, USA \\
p-AKT & $1: 500$ & 13038 & Cell Signaling Technology, Beverly, CA, USA \\
mTOR & $1: 500$ & $a b 2732$ & Abcam, Cambridge, MA, USA \\
PTEN & $1: 500$ & 9552 & Cell Signaling Technology, Beverly, CA, USA \\
Ub & $1: 500$ & 3933 & Cell Signaling Technology, Beverly, CA, USA \\
GAPDH & $1: 500$ & TA-08 & Zhongshanjingiao, Beijing, China \\
\hline
\end{tabular}

ACY1, aminoacylase 1; PI3K, phosphatidylinositol 3 kinase; AKT, protein kinase B; mTOR, mammalian target of rapamycin; PTEN, phosphatase and tensin homolog deleted on chromosome ten; GAPDH, glyceraldehyde-3-phosphate dehydrogenase.

was incubated with Dynabeads protein G for 1 hour, and incubated overnight at $4{ }^{\circ} \mathrm{C}$ with $2 \mu \mathrm{g}$ of antibody against PTEN, PI3K, AKT, and mTOR, which was followed by incubation with Dynabeads protein $G$ for 1 hour. The immune complex was then loaded onto gels using $\mathrm{Ub}$ antibody.

\section{MTT assay}

For the MTT assay, $3 \times 10^{3}$ cells (with $200 \mu \mathrm{L}$ growth medium) were seeded into 96-well plates overnight. Subsequently, MTT (Merck, Germany) was added and incubated at $37^{\circ} \mathrm{C}$ for 4 hours. After this, $150 \mu \mathrm{L}$ of dimethyl sulfoxide (DMSO) was added for 10 minutes. The absorbance was measured at $570 \mathrm{~nm}$.

\section{Flow cytometry assay}

Cells in different groups were seeded into 6-well plates $\left(5 \times 10^{5}\right)$. After 24 hours of treatment, cells were digested with $0.25 \%$ trypsin and resuspended in binding buffer. Following this, $5 \mu \mathrm{L}$ of Annexin V-FITC (Annexin V-FITC Apoptosis Detection kit, BD Biosciences, San Jose, CA, USA) and $5 \mu \mathrm{L}$ of propidium iodide (PI; Sigma-Aldrich, St. Louis, MO, USA) were added to each sample and incubated at $2-8{ }^{\circ} \mathrm{C}$ for 5 minutes in the dark. Fluorescence-activated apoptotic cell assays were conducted with a flow cytometer (BD FACSVantage SE, BD Biosciences Co., Franklin Lakes, NJ, USA). The sum of the data of Q2 and Q3 was the apoptosis rate (\%).

\section{Wound bealing assay}

NSCLC cells were seeded into 6 -well plates at a $2 \times 10^{5}$ cells/well density and transfected with sh-NC, sh-ACY1, and OE-NC or OE-ACY1. The scratch was made using a sterile $20-\mu \mathrm{L}$ pipette tip, and the medium was replaced with a serum-free medium until the cells reached a confluent monolayer. Cells were then incubated at $37^{\circ} \mathrm{C}$ for 24 hours. The cells were examined and photographed under phase contrast microscopy at 0 and 24 hours. The rate of cell mobility inhibition (\%) was calculated as follows: cell mobility $=$ scratch width $(24$ hours $) /$ scratch width (0 hour) $\times 100 \%$.

\section{Transwell chamber with Matrigel assay}

Cell invasion was detected by transwell chambers (8 $\mu \mathrm{m}$ pore size; BD Biosciences, San Diego, CA, USA) precoated with Matrigel (BD Biosciences). Next, $1.0 \times 10^{5}$ cells resuspended in $200 \mu \mathrm{L}$ of serum-free medium were inoculated into the upper chamber of the transwell. Meanwhile, $600 \mu \mathrm{L}$ of medium containing $15 \%$ FBS was added to the lower chamber. The cells were stained with $1 \%$ crystal violet for 20 minutes and then washed with PBS. The invaded cells were counted with a microscope after 48 hours.

\section{Xenotransplantation assay}

A total of 12 four-week-old male athymic BALB/C nude mice (Weitonglihua. Co., Ltd., Beijing, China) were used 
to assess cell tumorigenesis. The animals were kept in a professional laboratory for nude mice in an atmosphere of $24 \pm 1{ }^{\circ} \mathrm{C}$ with $60 \% \pm 5 \%$ relative humidity. A random number table method was used to divide the mice into 4 groups $(\mathrm{N}=3)$ : control, sh-ACY1, OE-ACY1, and OEACY1+OE-PTEN. In detail, $5 \times 10^{6} \mathrm{H} 1975$ cells with stable control, sh-ACY1, OE-ACY1, or OE-ACY1+OE-PTEN transfection were resuspended in $200 \mu \mathrm{L}$ PBS and then injected into the armpit area of the nude mice. Twentyeight days posttransplantation, the mice were sacrificed, and the tumors were collected for weighing. During the experiment, care was taken to minimize the pain of the mice without affecting the results of the experiment. No deaths occurred during the experiment. Euthanasia was administered when the diameter of the tumor was greater than $2 \mathrm{~cm}$ (not involved in this experiment). Protocols involving animals were approved by the ethical committee of Chongqing University Cancer Hospital \& Chongqing Cancer Institute \& Chongqing Cancer Hospital, and animal experiment was conducted in compliance with Chongqing University Cancer Hospital \& Chongqing Cancer Institute \& Chongqing Cancer Hospital guidelines for the care and use of animals.

\section{Statistical analysis}

Each measurement was obtained in 3 parallel tests. All experimental data are presented as the mean \pm SD. Statistical analyses were performed on GraphPad Prism 7 software with one-way analysis of variance followed by the Tukey's test for multiple groups and Student's $t$-test for 2 groups. $\chi^{2}$ test and Kaplan-Meier analysis were used to evaluate the clinical significance of ACY1 in NSCLC. Pearson Correlation Coefficient was used to verify the correlation between ACY1 and PTEN in NSCLC tissues. A $P$ value $<0.05$ was considered a statistically significant difference.

\section{Results}

\section{ACY1 expression was increased in NSCLC tissues and cells}

We first identified the expression patterns of ACY1 and PTEN in NSCLC tissues and cells. Increased ACY1 expression was observed in NSCLC tissues when compared to the adjacent normal tissues in both protein and mRNA levels, as determined by immunohistochemistry (Figure 1A), RT-PCR (Figure 1B), and western blotting (Figure 1C).
The expression of PTEN protein in NSCLC tissues was significantly lower than that of adjacent normal tissues (Figure 1C). In NSCLC tissues, the trends of PTEN and ACY1 were opposite. In addition, ACY1 and PTEN expression was also detected in NSCLC cell lines by realtime PCR (RT-PCR) and western blotting assays. ACY1 expression levels were increased in human NSCLC cell lines HCC2935, A549, and H1975 compared to those in human embryonic lung WI-38 cells, while PTEN mRNA and protein were decreased (Figure 1D,1E). These results suggest that ACY1 was deregulated in NSCLC.

\section{High ACY1 expression predicted an advanced clinical features and shorter overall survival for patients with NSCLC}

To investigate the role of ACY1 in NSCLC progression, we then assessed its clinical significance in NSCLC. A total of 91 patients with NSCLC were divided into 2 groups according to the expression levels of ACY1, among whom 60 had high ACY1 expression (high expression group) and 31 had low expression (low expression group). As shown in Table 3, there were no significant differences in gender, age, or histological type, but patients with ACY1 high expression had significantly lower differentiation $(\mathrm{P}=0.011)$, lymph node metastasis $(\mathrm{P}=0.002)$, and advanced tumor-node-metastasis (TNM) stage $(\mathrm{P}=0.006)$. Moreover, patients in the high expression group had shorter overall survival than those in the low expression group (Figure 1F). In addition, the expression of ACY 1 and PTEN in NSCLC tissues were negatively correlated (Figure 1G). These results suggest that ACY1 may have the potential to predict the clinical process and outcome of NSCLC patients.

\section{Overexpression of ACY1 facilitated NSCLC cell conversion to a malignant phenotype}

We investigated the effects of ACY1 in the progression of NSCLC in vitro via gain/loss-of-function assays. ACY1 expression was decreased to $20-30 \%$ when HCC2935 and H1975 cells were transfected with sh-2, while it was increased to $350-380 \%$ in mRNA levels and $200-210 \%$ in protein levels when cells were transfected with OEACY1 (Figure 2A,2B). Compared with controls, sh-ACY1 transfection significantly increased cell proliferation, and shACY1 impaired cell proliferation in HCC2935 (Figure 2C) and H1975 cells (Figure 2D). Cell apoptosis was decreased 

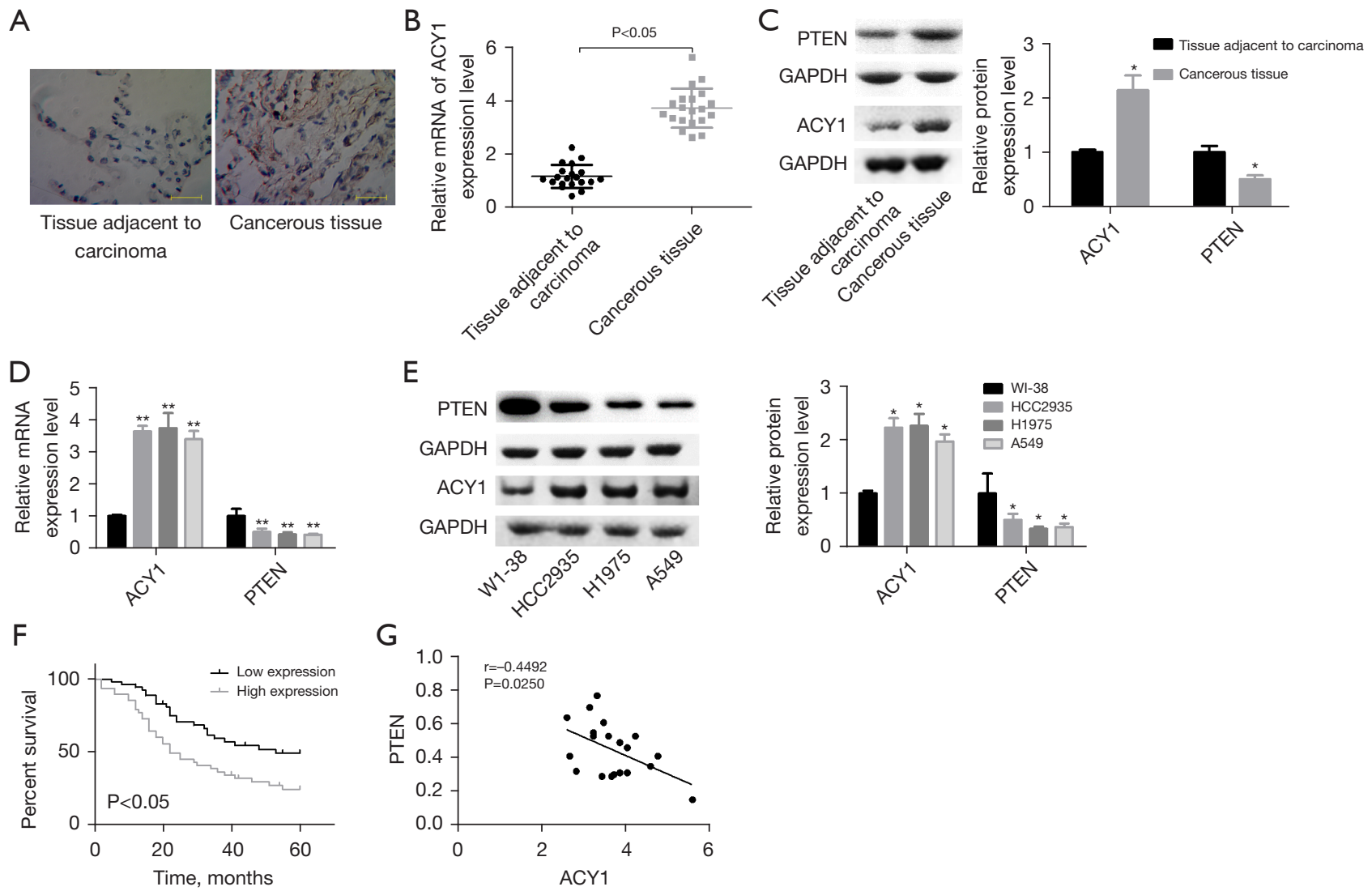

Figure 1 ACY1 expression was elevated in NSCLC tissue samples and cells. (A) Immunohistochemistry was used to assess ACY1 expression in lung tissues $(\times 400)$. (B) RT-PCR was performed to detect mRNA expression of ACY1 in NSCLC tissues and the adjacent normal tissues. (C) Western blotting assay was performed to detect the protein expressions of ACY1 and PTEN in NSCLC tissues and the adjacent normal tissues. (D,E) RT-PCR and western blotting assays were performed to detect the mRNA and protein expression of ACY1 and PTEN in human embryonic lung WI-38 cells and human NSCLC cell lines, HCC2935, A549, and H1975. (F) A survival curve was used to assess the influence of ACY1 expression on patients' overall survival. (G) Pearson correlation was used to verify the correlation between ACY1 and PTEN in NSCLC tissues (*, $\mathrm{P}<0.05$; **, $\mathrm{P}<0.01$ ). NSCLC, non-small cell lung cancer; ACY1, aminoacylase 1; RT-PCR, real-time polymerase chain reaction; PTEN, phosphatase and tensin homolog.

when HCC2935 and H1975 cells were transfected with OEACY1, and it was increased when ACY1 was downregulated (Figure 2E). In addition, ACY1 upregulation promoted cell migration and invasion, and knockdown of ACY1 caused the opposite to occur (Figure 3A-3C). We also detected the effects of ACY1 on epithelial-mesenchymal transition (EMT)-marked proteins. The results showed that when ACY1 was inhibited, the expression of E-cadherin protein increased, and the expression of $\mathrm{N}$-cadherin and Vimentin protein decreased (Figure 3D,3E). Overexpression of ACY1 produced the opposite result. Together, these findings demonstrated that ACY1 functioned as an oncogene in NSCLC.
Overexpression of ACY1 promoted the activation of PI3K/ AKT signaling through downregulation of PTEN

Subsequently, we investigated the effects of ACY1 expression on the activation of PI3K/AKT signaling. Compared with the control group, ACY1 upregulation significantly increased the protein expression of p-PI3K, p-AKT, and mTOR, whereas it reduced PTEN expression (Figure 4A) and showed no obvious influence in the mRNA levels of PI3K, AKT, mTOR, or PTEN (Figure 4B). To further explore the mechanism of ACY1 in regulating PTEN/PI3K/ AKT signaling, we used an IP assay to assess the interaction between ACY1 and PTEN/PI3K/AKT signaling-related proteins. The results showed that ACY1 could only combine 
Table 3 Association of ACY1 expression and the clinicopathologic features of patients with NSCLC

\begin{tabular}{|c|c|c|c|}
\hline \multirow{2}{*}{ Variable } & \multicolumn{2}{|c|}{ ACY1 expression } & \multirow{2}{*}{$P$ value } \\
\hline & High & Low & \\
\hline Gender & & & 0.649 \\
\hline Male & 37 & 21 & \\
\hline Female & 23 & 10 & \\
\hline Age (y) & & & 0.186 \\
\hline$<60$ & 38 & 15 & \\
\hline$\geq 60$ & 22 & 16 & \\
\hline Histological type & & & 0.512 \\
\hline Squamous cell carcinoma & 32 & 14 & \\
\hline Adenocarcinoma & 28 & 17 & \\
\hline Differentiation & & & 0.011 \\
\hline Well & 12 & 14 & \\
\hline Moderate & 22 & 12 & \\
\hline Poor & 26 & 5 & \\
\hline Lymph node metastasis & & & 0.002 \\
\hline No & 28 & 25 & \\
\hline Yes & 32 & 6 & \\
\hline TNM stage & & & 0.006 \\
\hline I & 10 & 11 & \\
\hline II & 17 & 14 & \\
\hline III & 33 & 6 & \\
\hline
\end{tabular}

NSCLC, non-small cell lung cancer; ACY1, aminoacylase 1; TNM, tumor-node-metastasis.

with PTEN, and this interaction was weakened when ACY1 was overexpressed in H1975 cells (Figure 4C). This might have been due to ACY1 inducing the degradation of PTEN protein. Moreover, ACY1 upregulation promoted the protein degradation of PTEN protein (Figure $5 A$ ), and increased the ubiquitination of PTEN protein (Figure $5 B$ ), and vice versa. Overall, these results demonstrated that ACY1 upregulation could promote PI3K/AKT signaling activation via downregulation of PTEN.

\section{ACY1 enbanced NSCLC cell tumorigenesis via downregulating PTEN}

We further examined the effects of ACY1/PTEN in the tumorigenesis of NSCLC cells. Tumorigenesis was significantly enhanced in $\mathrm{H} 1975$ cells during stable upregulation of ACY1, while it was decreased at the time of ACY1 downregulation. Overexpression of PTEN counteracted the enhancement of $\mathrm{H} 1975$ cell tumorigenesis induced by ACY1 upregulation (Figure $6 A, 6 B$ ), illustrating that $\mathrm{ACY} 1$ promoted cell tumorigenesis via downregulating PTEN in NSCLC.

\section{Discussion}

The American Joint Committee on Cancer (AJCC) $(20,21)$ has reported that up to $70 \%$ of NSCLC patients are incurable due to the extent of cancer at the time of diagnosis. A standard treatment for NSCLC, platinumbased chemotherapy, also shows low response rates of $17-32 \%$ and overall survival of 7.4-11.3 months for NSCLC patients at advanced stages (22). Therefore, it is crucial to further investigate the underlying mechanisms of NSCLC. This study focused on understanding the role and mechanism of ACY1, revealed the high fold change in the protein and mRNA expression levels of ACY1 in NSCLC tissues and cells, and showed that ACY1 has an oncogenic role in NSCLC. To our knowledge, there is a lack of studies on the clinical significance of ACY1 in NSCLC. However, the present study demonstrated that NSCLC patients with ACY1 high expression were prone to show low differentiation $(\mathrm{P}=0.011)$, lymph node metastasis $(\mathrm{P}=0.002)$, and advanced TNM stage $(\mathrm{P}=0.006)$, as well as poor prognosis, which was consistent with previous studies concerning the role of ACY1 in CRC (23).

ACY1 is commonly found in the kidneys and the brain. Its deregulation has been associated with autism and convulsions $(24,25)$. ACY1 has also been reported to play a role in the deacetylation of aflatoxin epoxy derivatives and thereby influence cell detoxification function (26). Moreover, studies have found ACY1 to be strongly implicated in carcinogenesis, with different roles in different types of cancers. For instance, low expression of ACY1 was found in HCC tissues, and ACY1 expression was significantly associated with tumor invasiveness; furthermore, overexpression of ACY1 in HCC BEL7402 cells was shown to significantly inhibit cell viability and invasiveness (9), revealing that ACY1 may function as a tumor suppressor. Conversely, ACY1 was reported to be overexpressed in CRC, and knockdown of ACY1 significantly inhibited cell proliferation and induced cell apoptosis $(10,11)$. In contrast to the ACY1 expression 

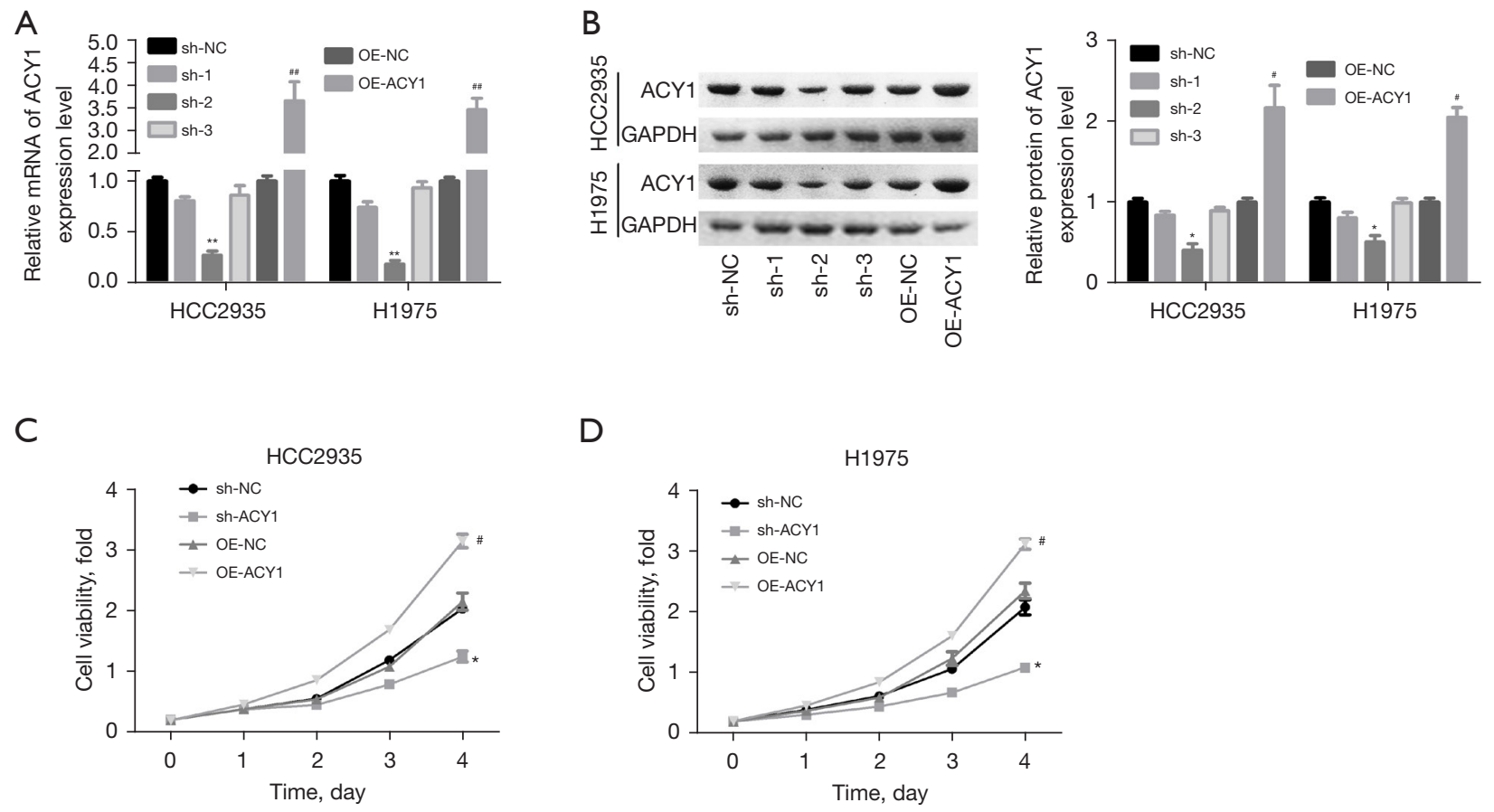

D

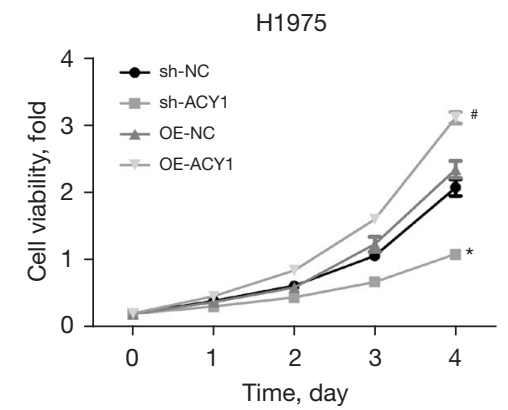

E
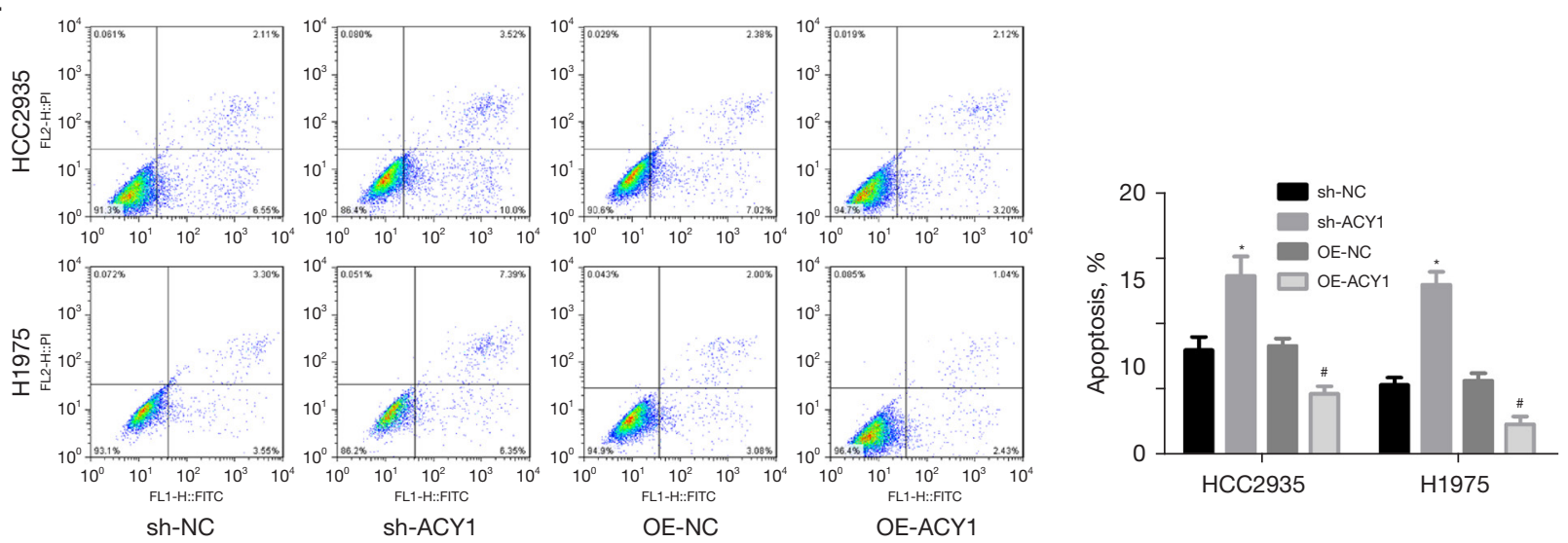

Figure 2 Role of altered expression of ACY1 in the proliferation and apoptosis of H1975 and HCC2935 cells. (A,B) RT-PCR and western blotting were applied to detect the mRNA and protein expressions after transfection with sh-ACY1, sh-NC, OE-NC, or OE-ACY1, untreated cells served as normal control. (C,D) MTT assay was performed to test cell growth. (E) Annexin V/PI double staining was used to assess cell apoptosis $\left({ }^{*}, \mathrm{P}<0.05,{ }^{* *}, \mathrm{P}<0.01\right.$, sh-2 or sh-ACY1 group versus sh-NC group; $, \mathrm{P}<0.05,{ }^{\# \#}, \mathrm{P}<0.01$, OE-ACY1 group versus $\mathrm{OE}-$ NC group). ACY1, aminoacylase 1.

profiles in SCLC (6), we found that ACY1 expression was obviously increased in NSCLC tissues and cells in both protein and mRNA levels. To measure the effects of this increase of ACY1 in NSCLC progression, gain/loss-offunction assays were performed in 2 NSCLC cell lines, H1975 and HCC2935. The results showed that cell growth, migration, invasion, and tumorigenesis were enhanced, while cell apoptosis was decreased when ACY1 was upregulated, indicating that ACY1 has a tumor-promoting role in NSCLC.

In addition, we investigated the mechanism of ACY1 in promoting the progression of NSCLC. We observed that ACY1 could activate PI3K/AKT signaling through downregulating PTEN. Studies have demonstrated that 

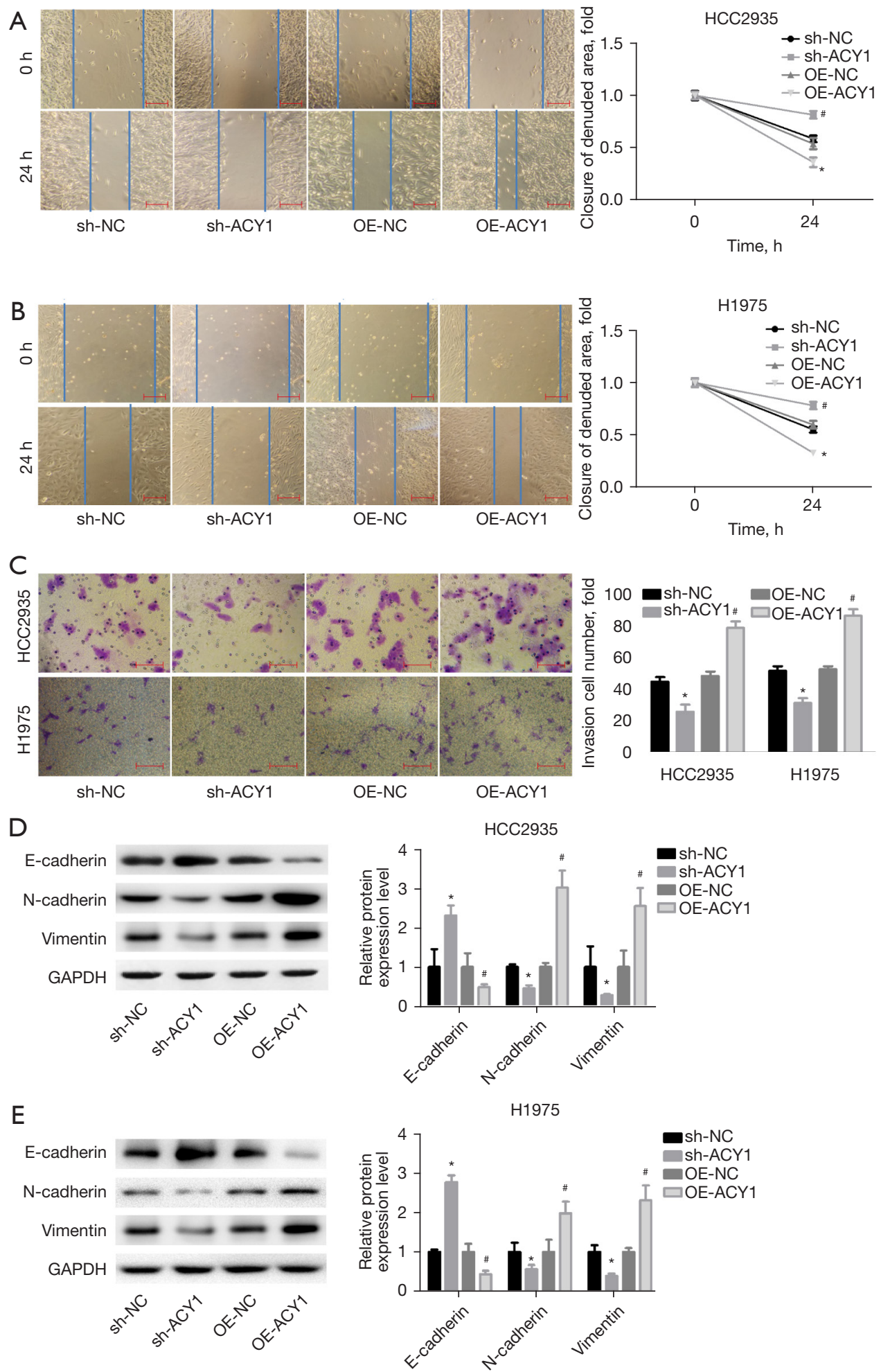

Figure 3 Role of altered expression of ACY1 in the migration and invasion of H1975 and HCC2935 cells. (A,B) Cell migration ability was assessed by wound healing assay after cell transfection $(\times 100)$. (C) Cell invasion ability was detected by Transwell assay, and the cells were stained by crystal violet $(\times 100)$. $(\mathrm{D}, \mathrm{E})$ Western blotting assay was performed to detect the EMT proteins. ${ }^{*}, \mathrm{P}<0.05$, sh-ACY1 group versus sh-NC group; \#, P<0.05, OE-ACY1 group versus OE-NC group). ACY1, aminoacylase 1; EMT, epithelial-mesenchymal transition. 

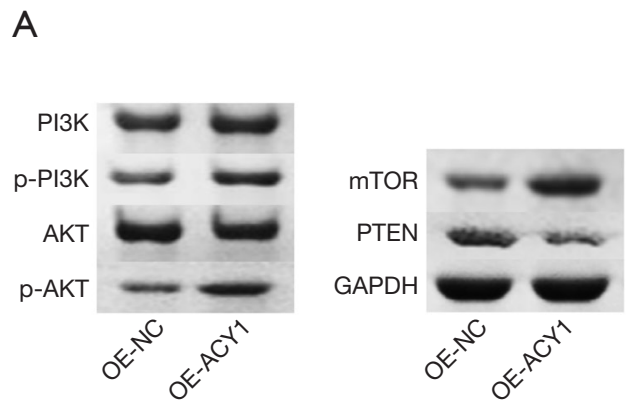

B

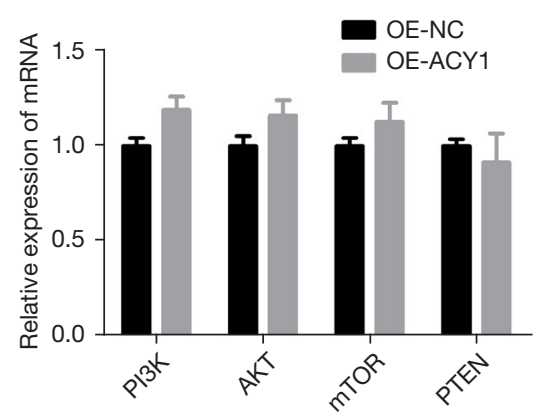

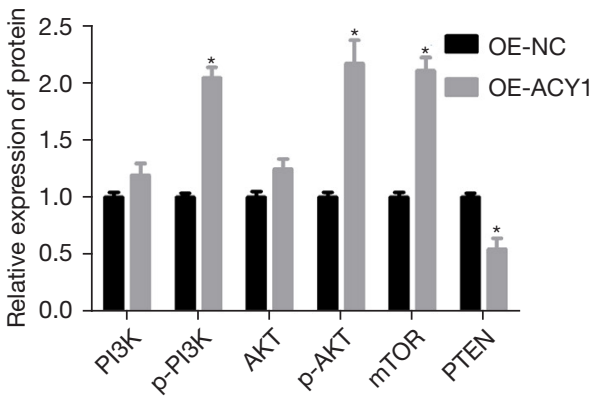

C

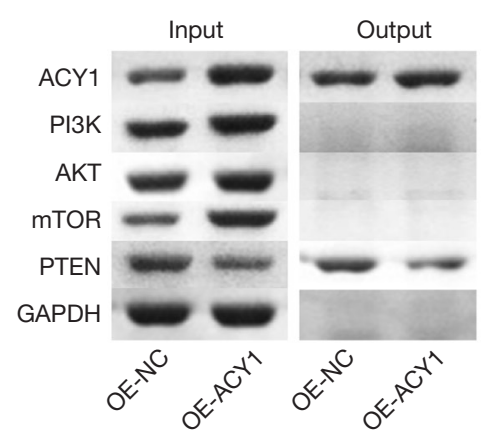

Figure 4 Role of ACY1 upregulation in the activation of PTEN/PI3K/AKT signaling. (A) Western blotting assay was used to detect the protein level of p-PI3K, PI3K, p-AKT, AKT, mTOR, and PTEN after 48 hours of cell transfection. (B) RT-PCR analysis of the mRNA level of PI3K, AKT, mTOR and PTEN after 24 hours of cell transfection. (C) IP assay was used to assess the crosstalk between ACY1 protein and PI3K, AKT, mTOR and PTEN proteins $(\mathrm{P}<0.05)$. $\left(^{*}, \mathrm{P}<0.05\right.$ vs. OE-NC group). ACY1, aminoacylase 1 ; RT-PCR, real-time polymerase chain reaction; PTEN, phosphatase and tensin homolog; IP, immunoprecipitation.

PTEN protein expression is significantly reduced in poorly differentiated NSCLC tumors compared to moderately differentiated NSCLC tumors. This is one of the main contributors to PI3K/AKT pathway activation and resistance to tyrosine kinase inhibitors (TKIs), including the most common chemotherapy drugs for NSCLC, such as gefitinib, erlotinib, and afatinib $(27,28)$. Furthermore, NSCLC patients with PTEN low expression level always have lower survival rates than those with PTEN high expression (29). This study, along with previous studies, illustrates the crucial role of PTEN in NSCLC. To further investigate the deregulation of PTEN and clarify the mechanism of ACY1 in NSCLC, we carried out western blotting and IP assays and found that ACY1 could interact with PTEN protein but not mTOR, AKT, or $\mathrm{PI} 3 \mathrm{~K}$, and could decrease its expression via accelerating its ubiquitination and degradation. Moreover, we observed that PTEN upregulation weakened the effect of ACY1 on tumorigenesis promotion in NSCLC H1975 cells, suggesting that ACY1 facilitated the progression of NSCLC through PTEN deletion. Although previous studies have reported that activation of ERK1/2 and expression of TGF- $\beta$ are implicated in ACY1-induced tumor cell proliferation $(9,30)$, the present study revealed, for the first time, that the PTEN/PI3K/AKT pathway may also be the target signaling of ACY1 in carcinogenesis. Based on this, we speculated that in NSCLC, the upregulation of ACY1 protein might activate the $\mathrm{PI} 3 \mathrm{~K} / \mathrm{AKT} / \mathrm{mTOR}$ pathway by inducing degradation of PTEN protein, thereby promoting cell proliferation and metastasis capabilities, and inhibiting apoptosis. However, the molecular mechanism underlying the manner in which ACY1 induces PTEN ubiquitination and degradation still needs to be explored by further indepth analysis.

In conclusion, this study suggests that ACY1 may function as an oncogene in NSCLC via regulating the PTEN/PI3K/AKT signaling pathway. It also demonstrates the clinical significance of ACY1 in NSCL, specifically, how ACY1 expression may be closely associated with an advanced clinical process and shorter overall survival for 
A

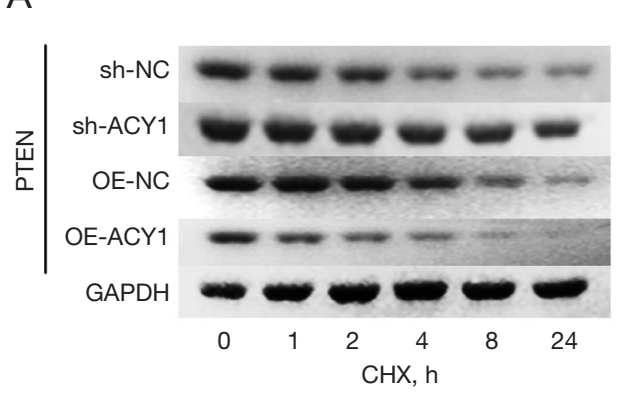

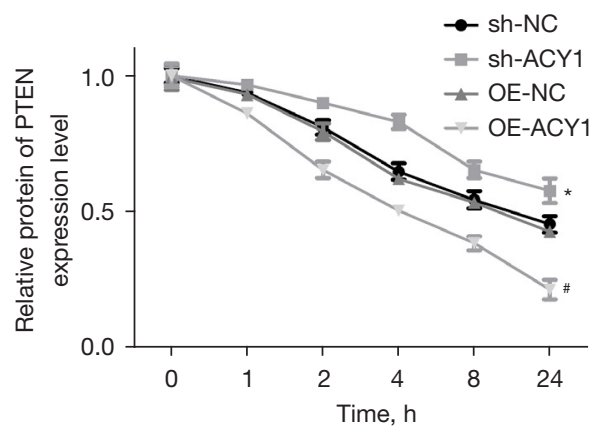

B

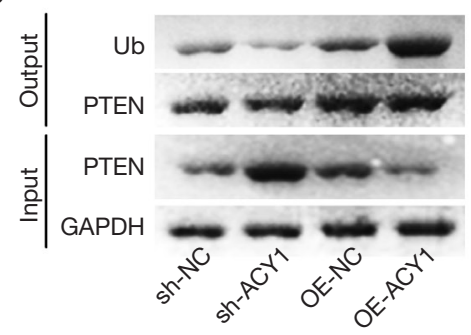

Figure 5 ACY1 overexpression promoted the ubiquitination degradation of PTEN protein. H1975 cells were transfected with OEACY1, OE-NC, sh-ACY1 or sh-NC for 24 hours, and then (A) $100 \mu \mathrm{g} / \mathrm{mL}$ of cycloheximide (CHX) was added to cell culture medium and incubated for $0,1,2,4,8$, and 24 hours to inhibit protein synthesis; subsequently, cells were collected for western blotting assay to assess PTEN expression (*, $\mathrm{P}<0.05$, sh-ACY1 group versus sh-NC group; ${ }^{*}, \mathrm{P}<0.05$, $\mathrm{OE}-\mathrm{ACY} 1$ group versus $\mathrm{OE}-\mathrm{NC}$ group). (B) IP assay was used to detect the interaction between PTEN and Ub proteins after 48 hours of cell transfection. ACY1, aminoacylase 1; PTEN, phosphatase and tensin homolog; IP, immunoprecipitation.

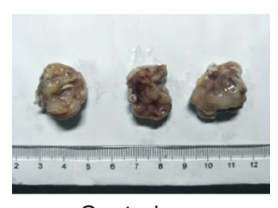

Control
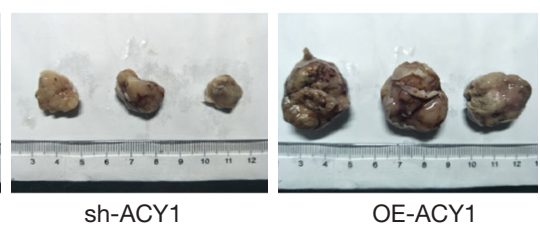

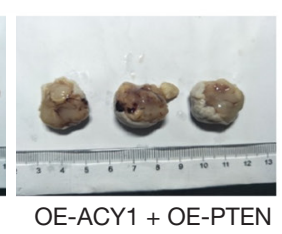

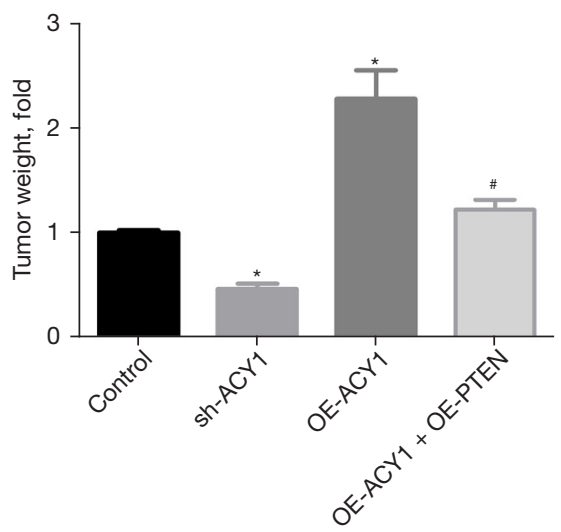

Figure 6 Evaluation of the effects of ACY1/PTEN in the tumorigenesis of H1975 cells. H1975 cells with ACY1 stable upregulation/ downregulation or ACY1 + PTEN stable upregulation were injected into the nude mice. Twenty-eight days after the injection, the tumors were extracted to measure the volume and weigh. (* $\mathrm{P}<0.05$, sh-ACY1/OE-ACY1 group versus control group; * $\mathrm{P}<0.05, \mathrm{OE}-\mathrm{ACY} 1+\mathrm{OE}-$ PTEN group versus OE-ACY1 group). ACY1, aminoacylase 1; PTEN, phosphatase and tensin homolog. 
patients with NSCLC. Our study aims to provide a better understanding of the pathogenesis and development of NSCLC. However, further exploration into the mechanism of ACY1 influence in the occurrence and progression of lung cancer is needed.

\section{Acknowledgments}

Funding: The study was funded in part by Chongqing University Cancer Hospital \& Chongqing Cancer Institute \& Chongqing Cancer Hospital (No. ZY201701001).

\section{Footnote}

Reporting Checklist: The authors have completed the ARRIVE reporting checklist. Available at https://dx.doi. org/10.21037/atm-21-3127

Data Sharing Statement: Available at https://dx.doi. org/10.21037/atm-21-3127

Conflicts of Interest: All authors have completed the ICMJE uniform disclosure form (available at https://dx.doi. org/10.21037/atm-21-3127). The authors have no conflicts of interest to declare.

Ethical Statement: The authors are accountable for all aspects of the work in ensuring that questions related to the accuracy or integrity of any part of the work are appropriately investigated and resolved. All procedures performed in this study involving human participants were in accordance with the Declaration of Helsinki (as revised in 2013). The study was approved by Ethics Committee of Chongqing University Cancer Hospital \& Chongqing Cancer Institute \& Chongqing Cancer Hospital (Chongqing, China) and informed consent was taken from all the patients. Protocols involving animals were approved by the ethical committee of Chongqing University Cancer Hospital \& Chongqing Cancer Institute \& Chongqing Cancer Hospital, and animal experiment was conducted in compliance with Chongqing University Cancer Hospital \& Chongqing Cancer Institute \& Chongqing Cancer Hospital guidelines for the care and use of animals.

Open Access Statement: This is an Open Access article distributed in accordance with the Creative Commons Attribution-NonCommercial-NoDerivs 4.0 International License (CC BY-NC-ND 4.0), which permits the non- commercial replication and distribution of the article with the strict proviso that no changes or edits are made and the original work is properly cited (including links to both the formal publication through the relevant DOI and the license). See: https://creativecommons.org/licenses/by-nc-nd/4.0/.

\section{References}

1. Dempke WC. Targeted Therapy for NSCLC--A Doubleedged Sword? Anticancer Res 2015;35:2503-12.

2. Ettinger DS, Wood DE, Akerley W, et al. Non-Small Cell Lung Cancer, Version 6.2015. J Natl Compr Canc Netw 2015;13:515-24.

3. Jiao Z, Yu A, He X, et al. Bioinformatics analysis to determine the prognostic value and prospective pathway signaling of miR-126 in non-small cell lung cancer. Ann Transl Med 2020;8:1639.

4. Wu KL, Tsai MJ, Yang CJ, et al. Liver metastasis predicts poorer prognosis in stage IV lung adenocarcinoma patients receiving first-line gefitinib. Lung Cancer 2015;88:187-94.

5. Heese D, Löffler HG, Röhm KH. Further characterization of porcine kidney aminoacylase I reveals close similarity to 'renal dipeptidase'. Biol Chem Hoppe Seyler 1988;369:559-66.

6. Miller YE, Minna JD, Gazdar AF. Lack of expression of aminoacylase-1 in small cell lung cancer. Evidence for inactivation of genes encoded by chromosome 3p. J Clin Invest 1989;83:2120-4.

7. Balabanov S, Zimmermann U, Protzel C, et al. Tumourrelated enzyme alterations in the clear cell type of human renal cell carcinoma identified by two-dimensional gel electrophoresis. Eur J Biochem 2001;268:5977-80.

8. Hwa JS, Park HJ, Jung JH, et al. Identification of proteins differentially expressed in the conventional renal cell carcinoma by proteomic analysis. J Korean Med Sci 2005;20:450-5.

9. Wei X, Li J, Xie H, et al. Proteomics-based identification of the tumor suppressor role of aminoacylase 1 in hepatocellular carcinoma. Cancer Lett 2014;351:117-25.

10. Shi H, Hayes MT, Kirana C, et al. Overexpression of aminoacylase 1 is associated with colorectal cancer progression. Hum Pathol 2013;44:1089-97.

11. Shi H, Hood KA, Hayes MT, et al. Proteomic analysis of advanced colorectal cancer by laser capture microdissection and two-dimensional difference gel electrophoresis. J Proteomics 2011;75:339-51.

12. Chen Z, Gao J, Sun J, et al. Aminoacylase 1 (ACY1) Mediates the Proliferation and Migration of 
Neuroblastoma Cells in Humans Through the ERK/ Transforming Growth Factor $\beta$ (TGF- $\beta$ ) Signaling Pathways. Med Sci Monit 2021;27:e928813.

13. Ersahin T, Tuncbag N, Cetin-Atalay R. The PI3K/AKT/ mTOR interactive pathway. Mol Biosyst 2015;11:1946-54.

14. Fan X, Huang H, Ji Z, et al. Long non-coding RNA MEG3 functions as a competing endogenous RNA of miR-93 to regulate bladder cancer progression via $\mathrm{PI} 3 \mathrm{~K} /$ AKT/mTOR pathway. Transl Cancer Res 2020;9:1678-88.

15. Pérez-Ramírez C, Cañadas-Garre M, Molina MÁ, et al. PTEN and PI3K/AKT in non-small-cell lung cancer. Pharmacogenomics 2015;16:1843-62.

16. Xia M, Tong JH, Ji NN, et al. Tramadol regulates proliferation, migration and invasion via PTEN/PI3K/ AKT signaling in lung adenocarcinoma cells. Eur Rev Med Pharmacol Sci 2016;20:2573-80.

17. Cao L, Chen J, Ou B, et al. GAS5 knockdown reduces the chemo-sensitivity of non-small cell lung cancer (NSCLC) cell to cisplatin (DDP) through regulating miR-21/PTEN axis. Biomed Pharmacother 2017;93:570-9.

18. Shen H, Guan D, Shen J, et al. TGF- $\beta 1$ induces erlotinib resistance in non-small cell lung cancer by downregulating PTEN. Biomed Pharmacother 2016;77:1-6.

19. Xiao H, Liu Y, Liang P, et al. TP53TG1 enhances cisplatin sensitivity of non-small cell lung cancer cells through regulating miR-18a/PTEN axis. Cell Biosci 2018;8:23.

20. Yu Y, Zhang P, Yao R, et al. Prognostic value of log odds of positive lymph nodes in node-positive lung squamous cell carcinoma patients after surgery: a SEER populationbased study. Transl Lung Cancer Res 2020;9:1285-301.

21. Edge SB, Compton CC. The American Joint Committee on Cancer: the 7th edition of the AJCC cancer staging manual and the future of TNM. Ann Surg Oncol 2010;17:1471-4.

22. Kelly K, Crowley J, Bunn PA Jr, et al. Randomized phase III trial of paclitaxel plus carboplatin versus vinorelbine

Cite this article as: Chen $\mathrm{H}$, Wang W, Xiao C, Xia D, Li F, Liu S. ACY1 regulating PTEN/PI3K/AKT signaling in the promotion of non-small cell lung cancer progression. Ann Transl Med 2021;9(17):1378. doi: 10.21037/atm-21-3127 plus cisplatin in the treatment of patients with advanced non--small-cell lung cancer: a Southwest Oncology Group trial. J Clin Oncol 2001;19:3210-8.

23. Yu B, Liu X, Cao X, et al. Study of the expression and function of ACY1 in patients with colorectal cancer. Oncol Lett 2017;13:2459-64.

24. Smith T, Ghandour MS, Wood PL. Detection of N-acetyl methionine in human and murine brain and neuronal and glial derived cell lines. J Neurochem 2011;118:187-94.

25. Tylki-Szymanska A, Gradowska W, Sommer A, et al. Aminoacylase 1 deficiency associated with autistic behavior. J Inherit Metab Dis 2010;33 Suppl 3:S211-4.

26. Stocker P, Brunel JM, de Rezende L, et al. Aminoacylase 1-catalysed deacetylation of bioactives epoxides mycotoxinderived mercapturates; 3,4-epoxyprecocenes as models of cytotoxic epoxides. Biochimie 2012;94:1668-75.

27. Jin G, Kim MJ, Jeon HS, et al. PTEN mutations and relationship to EGFR, ERBB2, KRAS, and TP53 mutations in non-small cell lung cancers. Lung Cancer 2010;69:279-83.

28. Spoerke JM, O'Brien C, Huw L, et al. Phosphoinositide 3-kinase (PI3K) pathway alterations are associated with histologic subtypes and are predictive of sensitivity to PI3K inhibitors in lung cancer preclinical models. Clin Cancer Res 2012;18:6771-83.

29. Endoh H, Yatabe Y, Kosaka T, et al. PTEN and PIK3CA expression is associated with prolonged survival after gefitinib treatment in EGFR-mutated lung cancer patients. J Thorac Oncol 2006;1:629-34.

30. Yijing L, Liu H, Yuan C, et al. The effects of qindancapsule-containing serum on the TGF- $\beta 1 / \mathrm{ERK}$ signaling pathway, matrix metalloproteinase synthesis and cell function in adventitial fibroblasts. Pharm Biol 2013;51:712-21.

(English Language Editors: G. Stone and J. Gray) 